\title{
Tenascin XB Is a Novel Diagnostic Marker for Malignant Mesothelioma
}

\author{
KOJI NAKAYAMA ${ }^{1}$, MASAHIRO SEIKE ${ }^{1}$, RINTARO NORO ${ }^{1}$, SUSUMU TAKEUCHI ${ }^{1}$, \\ KUNIKO MATSUDA $^{1}$, SHINOBU KUNUGI $^{2}$, KAORU KUBOTA ${ }^{1}$ and AKIHIKO GEMMA ${ }^{1}$ \\ ${ }^{1}$ Division of Pulmonary Medicine and Oncology, Graduate School of Medicine, \\ Nippon Medical School, Tokyo, Japan; \\ ${ }^{2}$ Division of Pathology, Graduate School of Medicine, Nippon Medical School, Tokyo, Japan
}

\begin{abstract}
Background/Aim: Malignant mesothelioma (MM) is an aggressive tumor with poor prognosis. The establishment of a new diagnostic and therapeutic approach for $M M$ is expected. This study investigated the diagnostic significance of tenascin XB (TNXB) for MM. Materials and Methods: $T N X B$ gene expression was found to be significantly higher in MM tumor tissues compared to paired normal tissues, as assessed by the Gene Expression Omnibus database. The inhibition of TNXB using small interfering RNAs suppressed the proliferation and colony formation of $M M$ cells. Expression of TNXB and calretinin, a current diagnostic marker of MM, was evaluated by immunohistochemistry. Results: The sensitivity and specificity of TNXB for MM were $80.0 \%$ and $69.5 \%$, respectively. When the detection of TNXB was combined with that of calretinin, $83.3 \%$ of MM cases were detected. Conclusion: These findings suggest that TNXB is a novel diagnostic biomarker for MM. A combination of detecting TNXB and calretinin may be useful for the differential diagnosis of MM from lung adenocarcinoma.
\end{abstract}

Malignant mesothelioma (MM) is a highly aggressive and fatal neoplasm. The prognosis remains unfavorable and the median survival after diagnosis is $9-12$ months $(1,2)$. MM is typically associated with exposure to asbestos (3). The latency period of MM development is some decades from asbestos exposure. The number of patients with MM is forecasted to steadily increase because of past occupational asbestos exposure. In early stages of MM, surgical resection should be considered. However, it is difficult to completely remove MM because of invasion and

Correspondence to: Masahiro Seike, MD, Ph.D., Division of Pulmonary Medicine and Oncology, Graduate School of Medicine, Nippon Medical School, Bunkyo-ku, Tokyo, 113-8063, Japan. E-mail: mseike@nms.ac.jp

Key Words: Tenascin XB, malignant mesothelioma, diagnostic marker. metastasis. Standard chemotherapy with cisplatin and pemetrexed is recommended as first-line chemotherapy (4). The immune-check point inhibitor nivolumab is also approved for use as second-line therapy (5). However, the prognosis of MM is still unsatisfactory. Furthermore, the pathological diagnosis of MM is difficult, especially its differentiation from lung adenocarcinoma. Therefore, novel diagnostic and therapeutic strategies need to be established for MM.

We previously identified 18 transcripts, including osteopontin, that correlated with susceptibility to pemetrexed (6). Three of these 18 transcripts were identified as deriving from tenascin $\mathrm{X}-\mathrm{A}$ and $-\mathrm{B}(T N X \mathrm{~A} / T N X B)$ genes. Tenascin is an extracellular matrix protein glycoprotein. TNX was reported as a differential diagnostic marker between MM and metastatic carcinoma, as well as between MM and ovarian carcinoma or primary peritoneal carcinoma (7). TNXA is a duplicated section of $T N X B$ and is probably a pseudogene; however, the function and clinical significance of TNXB in MM has not been clarified yet. In this study, we investigated the potential diagnostic significance and therapeutic use of TNXB in MM.

\section{Materials and Methods}

TNXB gene expression analysis. Gene expression data in the GSE51024 study from the Gene Expression Omnibus (GEO) database was used (8). TNXB gene expression of malignant pleural mesothelioma (MPM) and paired normal tissues by Affymetrix U133 plus 2.0 chips was statistically analyzed (8).

Cell culture. Five human MM cell lines were used in this study. The normal mesothelium cell line Met5A was used as a standard control. NCI-H28, NCI-H2452, NCI-H2052, NCI-H226 and Met5A were purchased from the American Type Culture Collection (ATCC, Manassas, VA, USA). ACC-MESO4 was obtained from the Riken Cell Bank (Tsukuba, Japan) (9). Five human lung adenocarcinoma cell lines: A549, NCI-H441, HCC-827 with epidermal growth factor receptor $(E G F R)$ exon 19 deletion and NCI-H1975 with EGFR exon 20 T790M mutation were acquired from the ATCC. LC2/ad was obtained from the European Collection of Authenticated Cell Cultures (Salisbury, UK). These cells were maintained in RPMI- 
1640 medium (Gibco-BRL, Carlsbad, CA, USA) supplemented with penicillin, streptomycin, and $10 \%$ fetal bovine serum at $37^{\circ} \mathrm{C}$ in an incubator with $5 \%$ carbon dioxide. All cell lines were routinely screened for the presence of mycoplasma by MycoAlert ${ }^{\mathrm{TM}}$ Mycoplasma Detection Kit (Lonza, Geneva, Switzerland).

RNA isolation and quantitative real-time polymerase chain reaction (PCR) analysis. Total messenger RNA was extracted from cells with TRIzol reagent (Thermo Fisher Scientific (Waltham, MA, USA) or ISOGEN (Nippongene, Tokyo, Japan) as previously described $(10,11)$. cDNA was synthesized from $1 \mu \mathrm{g}$ of total RNA using Superscript Reverse Transcriptase (Thermo Fisher Scientific). Quantitative real-time PCR assay was performed using the TaqMan probe Human Gene Expression Assay (Thermo Fisher Scientific). The TNXB probe was Hs00372889_g1. Glyceraldehyde 3-phosphate dehydrogenase $(G A D P H)$ was used as a normal control (Hs02786624_g1). Gene expression was quantified as the $2^{-\Delta \Delta \mathrm{Ct}}$ value (12).

Oligonucleotide transfection. Gene silencing was performed by small interfering RNAs (siRNAs) purchased from Thermo Fisher Scientific. The predesigned siRNA targeting TNXB was s14302. Silencer Select Negative Control \#1 siRNA was used as a negative control (Thermo Fisher Scientific). H2452 and H226 cells were seeded in a 6-well plate or $100 \mathrm{~mm}$ dish and transfected with siRNA using Lipofectamine RNAiMAX reagent (Thermo Fisher Scientific) for 74 hours. The final concentration of siRNA was $25 \mathrm{nM}$.

Proliferation assay. Overall, 2,000 H2452 and H226 cells per well were plated in a 96 -well plate. Cell proliferation was quantified by MTS assay using an absorbance reader. The absorbance measurement was performed every day. Cultures were grown for 3 or 4 days. Cell growth was assessed by the absorbance rate relative to the initial day's level.

Colony formation assay. Overall, $350 \mathrm{H} 2452$ and $\mathrm{H} 226$ cells were seeded in a 6-well plate and incubated for 2 weeks and 3 weeks, respectively. Then the medium was aspirated. The wells were washed with phosphate-buffered saline and fixed with $99.8 \%$ methanol for $15 \mathrm{~min}$. After staining with Giemsa for 15 minutes, the number of colonies was counted. A colony was defined as consisting of more than 50 cells.

Immunohistochemistry. TNXB and calretinin protein expression was evaluated by immunohistochemistry (IHC) using MM and lung adenocarcinoma tissue microarrays (TMA), MS801a and HLugA150CS02, respectively (US Biomax, Rockville, MD, USA). MS801a was composed of $30 \mathrm{MM}$ cases and HLugA150CS02 included 75 lung adenocarcinoma tissues. Six bronchioalveolar carcinoma samples were excluded. Overall, $30 \mathrm{MM}$ samples and 69 lung adenocarcinoma samples were finally evaluated. Slides were stained overnight at $4{ }^{\circ} \mathrm{C}$ with rabbit polyclonal antibody to human TNXB (ab198871; Abcam, Cambridge, MA, USA) at a final dilution of 1:50. Rabbit monoclonal antibody to human calretinin (MA5-14540) was used at a final dilution of 1:100 (Thermo Fisher Scientific). The slides were incubated with antirabbit IgG (BA-1000, Vector Labs, Burlingame, CA, USA) as a secondary antibody and the avidin-biotin complex method was performed as previously described (13). Images were captured by
Table I. Numbers of tenascin XB (TNXB)- and calretinin-positive and negative cases by immunohistochemistry.

Calretinin, $\mathrm{n}$

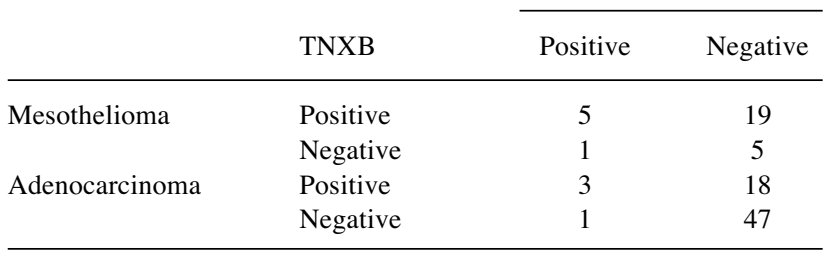

Table II. Sensitivity and specificity when using tenascin XB (TNXB), calretinin, and their combination as diagnostic markers for malignant mesothelioma.

\begin{tabular}{lcc}
\hline Marker & Sensitivity & Specificity \\
\hline TNXB & $80.0 \%$ & $69.5 \%$ \\
Calretinin & $20.0 \%$ & $94.2 \%$ \\
TNXB+Calretinin & $83.3 \%$ & $68.1 \%$ \\
\hline
\end{tabular}

AX80 optical microscope (Olympus, Tokyo, Japan). Stained areas were measured by the LuminaVision program (MITANI Corporation, Tokyo, Japan) using three independent views of every sample. The cut-off point for the proportion of stained area was determined using the mean of three measurements by receiver operating characteristic (ROC) curves.

Statistical analysis. Continuous variable data are expressed by the mean and standard error (SE). Paired samples of MM and normal lung parenchyma in the dataset were compared by the Wilcoxon signed-rank test. Other data between two independent groups was evaluated by the Mann-Whitney $U$-test. All $p$-values were twosided, and the significance level was set at less than 0.05. All statistical analyses were performed by JMP software (SAS Institute Japan, Tokyo, Japan)

\section{Results}

We evaluated the TNXB gene expression of malignant pleural mesothelioma (MPM) patients in the GSE51024 study from the Gene Expression Omnibus database (8). Figure 1A shows the $T N X B$ gene expression level in MPM and paired normal tissues $(\mathrm{n}=41) . T N X B$ gene expression levels were found to be significantly higher in MPM tissues than in paired normal tissues $(p<0.001)(8)$. In the present study, we also evaluated $T N X B$ expression using MM and lung adenocarcinoma cell lines. High $T N X B$ expression was observed in three out of five MM cell lines. In contrast, five lung adenocarcinoma cell lines showed lower expression than Met5A (Figure 1B). These results suggest that high $T N X B$ expression may contribute to the carcinogenesis of MM. 


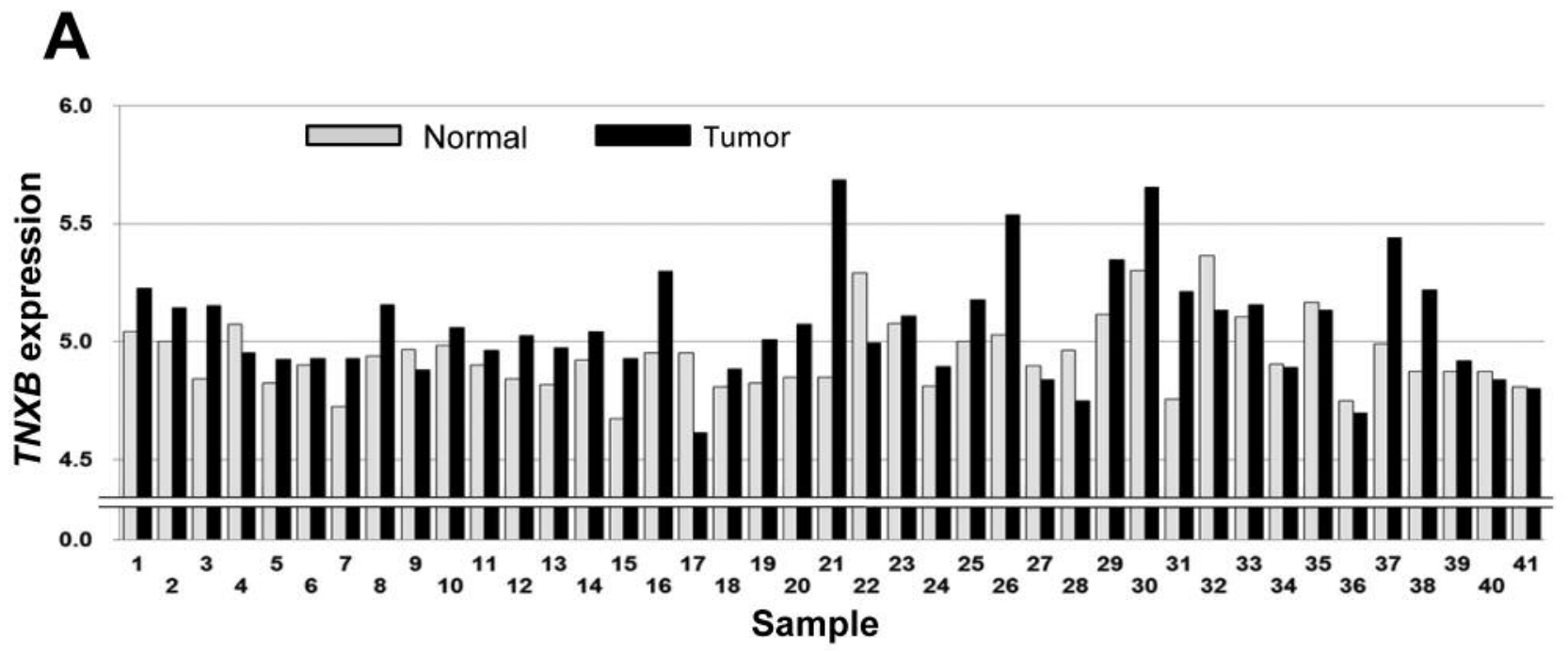

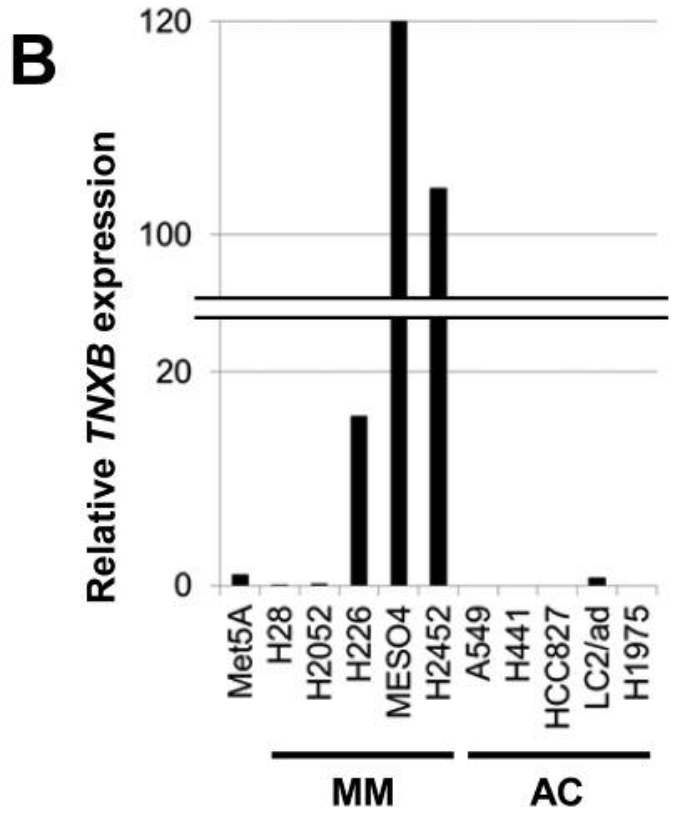

Next, in order to clarify the significance of $T N X B$ overexpression in MM, we evaluated whether $T N X B$ inhibition affected the proliferation and colony formation abilities of MM. Figure 2A shows the successful suppression of TNXB in H2452 and H226 mesothelioma cells using siRNA transfection. After $T N X B$ inhibition, proliferation was dramatically reduced on day 3 in $\mathrm{H} 2452$ and $\mathrm{H} 226$ cells (Figure 2B). A colony formation assay was also performed on H2452 and H226 cells after TNXB siRNA treatment. After the suppression of $T N X B$, the colony formation ability was significantly repressed in $\mathrm{H} 2452$ cells $(p<0.05)$, and showed a tendency to be repressed in $\mathrm{H} 226$ cells $(p=0.12)$ (Figure $2 \mathrm{C}$
Figure 1. A: Tenascin XB (TNXB) gene expression level of malignant pleural mesothelioma (MPM; black bars) and paired normal tissues (gray bars) (N=41) by Affymetrix U133 plus 2.0 chips. A set of adjoining black and gray bars represents a single individual. TNXB gene expression levels were found to be significantly higher in MPM tissues than in paired normal tissues $(p<0.001)$. B: Relative TNXB expression levels were compared between mesothelioma (MM) and lung adenocarcinoma $(A C)$ cell lines.

and D). These results suggest that $T N X B$ is involved in the carcinogenesis and progression of MM.

Finally, we examined TNXB protein level in MM and lung adenocarcinoma tissues from TMAs by IHC. Figure 3A shows representative positive and negative TNXB expression in MM and lung adenocarcinoma. The area positively stained for TNXB was significantly higher in MM than in adenocarcinoma tissues $(p<0.001)$ (Figure 3B).

An ROC curve for TNXB staining was generated and evaluated to differentiate MM from lung adenocarcinoma. The area under the curve (AUC) was 0.718 (Figure 3C). When a positively stained area of $1.4 \%$ was established as the cut-off, 24 out of $30 \mathrm{MM}$ cases and 21 out of 69 lung adenocarcinoma cases were TNXB-positive (Figure 3D) (Table I). The sensitivity was $80.0 \%$ and the specificity was $69.5 \%$ (Table II).

Calretinin is widely used in clinical settings as a diagnostic marker for MM. Therefore, we also conducted calretinin IHC staining in serial sections of TMA slides. Figure 3A shows representative positive and negative calretinin staining. When the calretinin-stained area was more than $0.80 \%$, tissues tended to be judged visually as positive. As shown in Table I, 6 out of $30 \mathrm{MM}$ cases had positive calretinin staining using TMA MS801a. In contrast, only four out of 69 adenocarcinoma cases were positive. The sensitivity and specificity for calretinin staining were $20.0 \%$ and $94.2 \%$, respectively (Table II). Interestingly, TNXB was positive in 19 

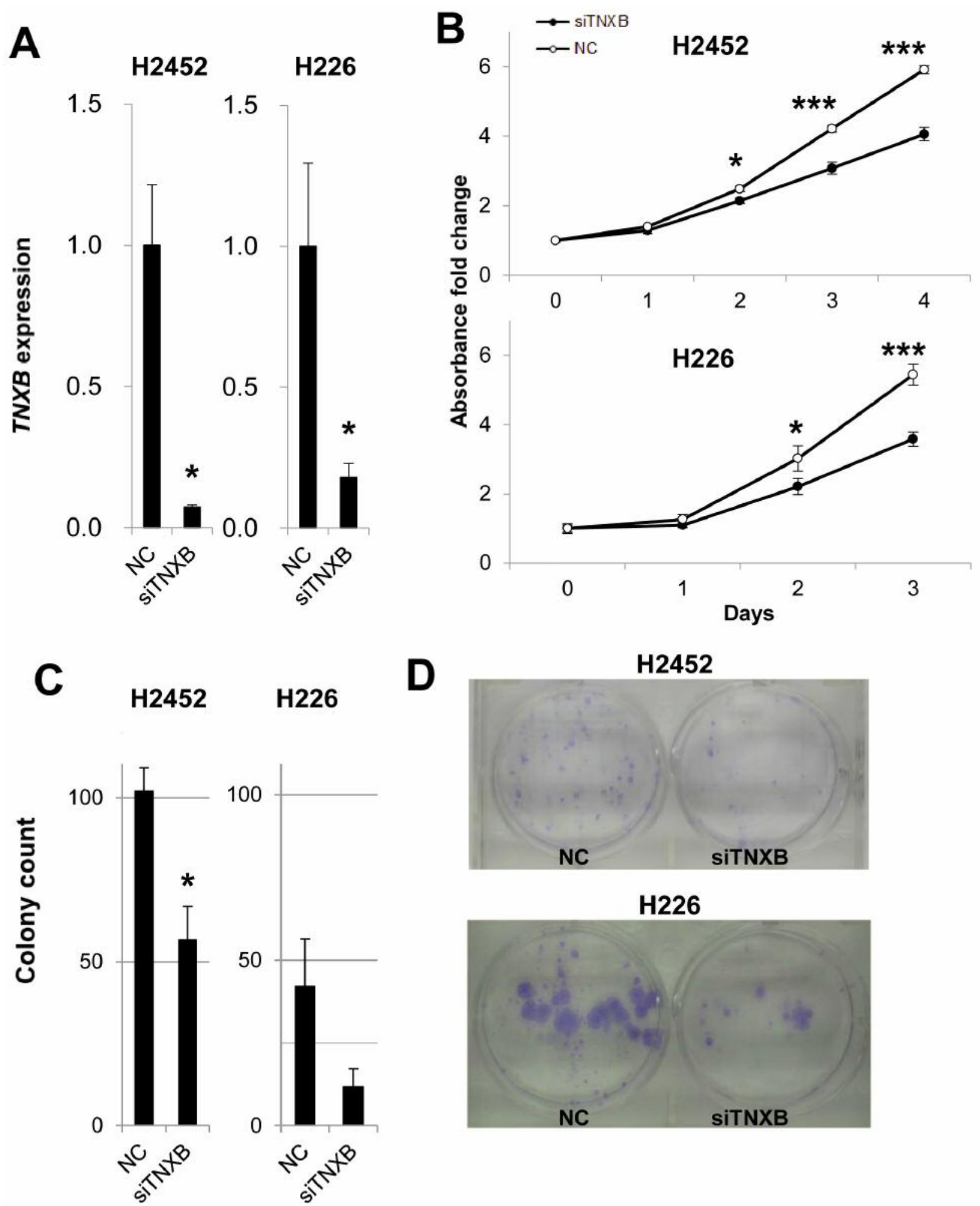

\section{H226}

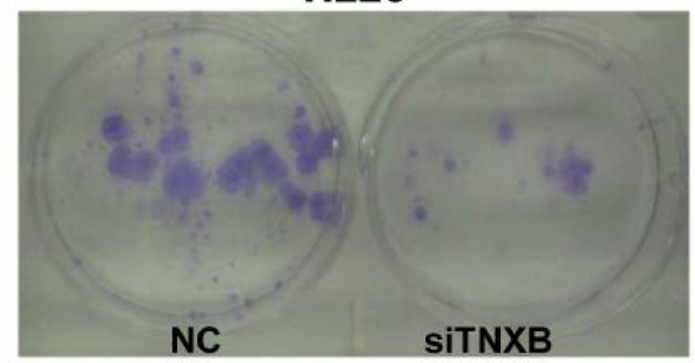

Figure 2. A: Tenascin XB (TNXB) gene expression in $H 2452$ and H226 cells after treatment with small interfering RNA against TNXB (siTNXB) and in negative control $(N C)$ cells. siTNXB significantly suppressed TNXB expression $\left({ }^{*} p<0.05\right)$. B: Cell growth of H2452 and H226 cells significantly decreased after siTNXB treatment at $* p<0.05$ and $* * * p<0.0001$. C: Colony formation after siTNXB treatment significantly decreased in $H 2452$ and $H 226$ cells $(* p<0.05)$. D: Representative images of plates after siTNXB treatment of H2452 and H226 cells. Data show the mean \pm SE. Proliferation and colony formation assay were performed independently three times. 
A

TNXB
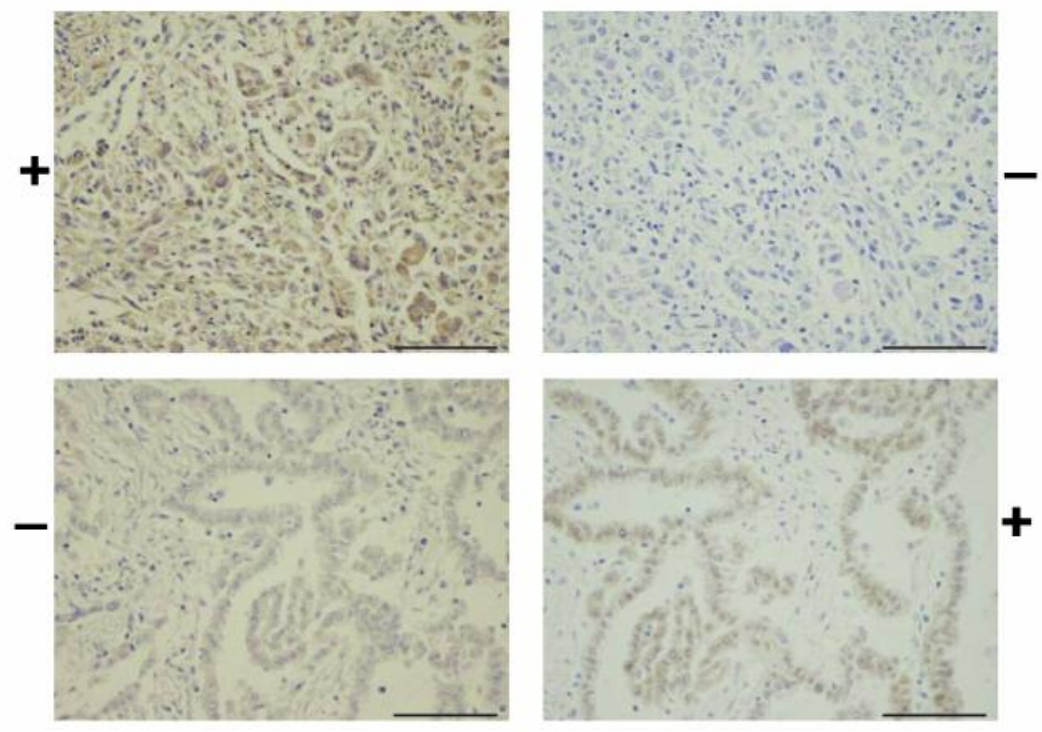

$\mathbf{C}$

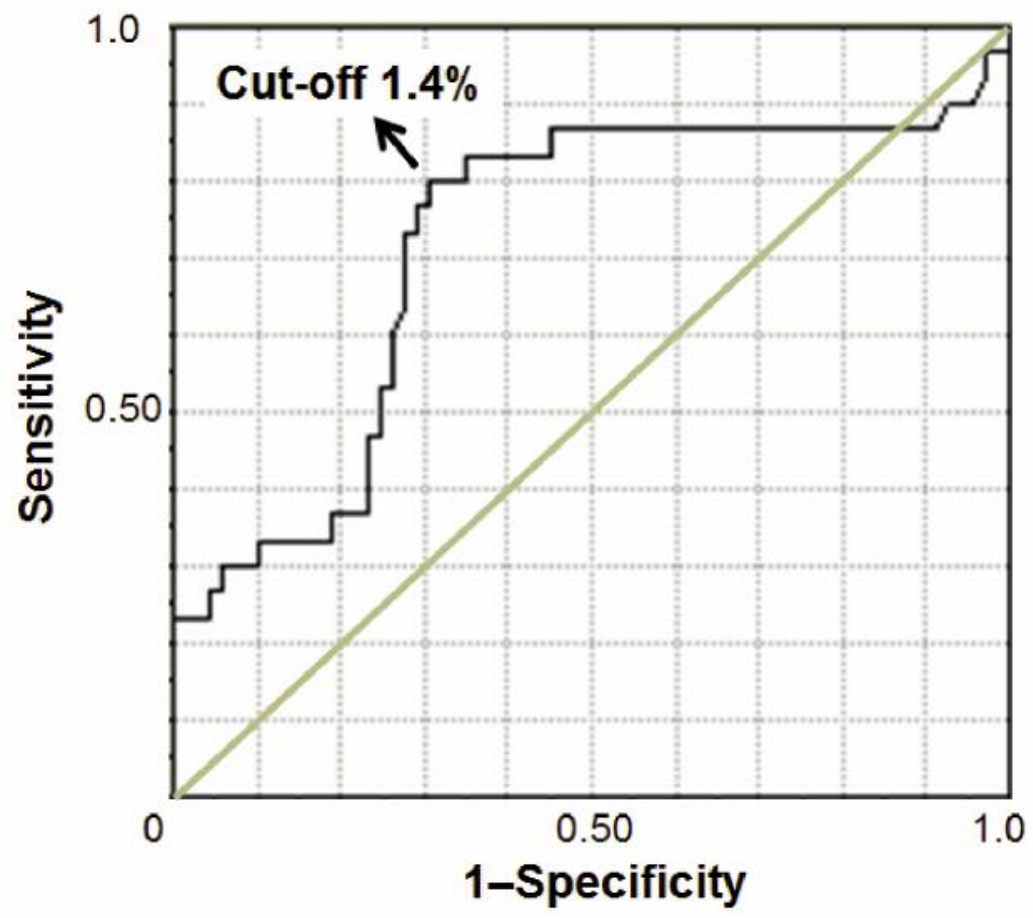

B

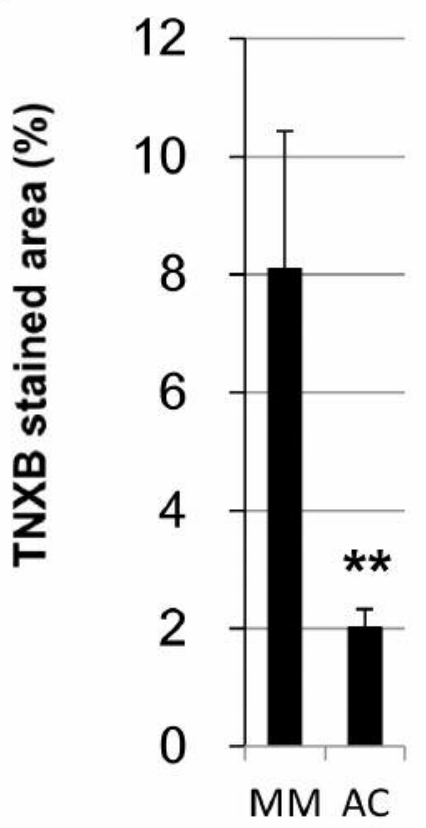

D
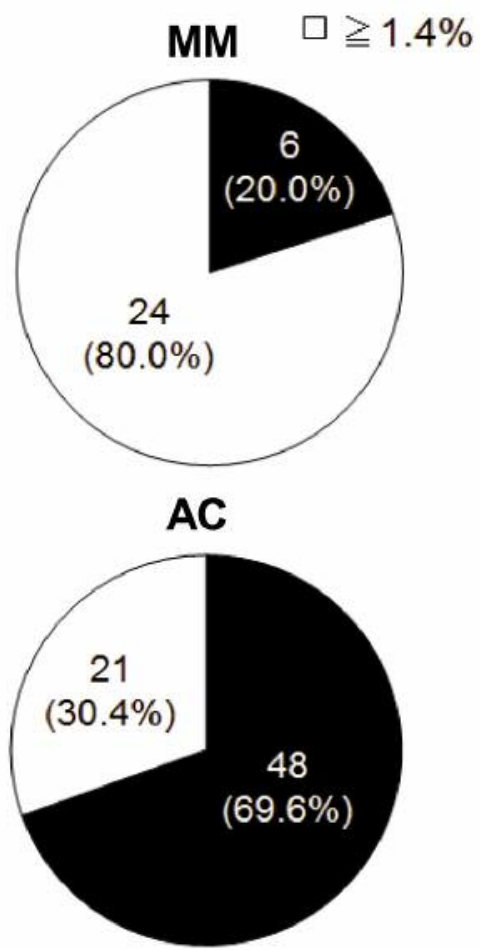

Figure 3. Tenascin XB (TNXB) and calretinin protein expression in malignant mesothelioma (MM) and lung adenocarcinoma (AC) tissues by immunohistochemistry. A: Representative cases from MM are shown in the upper and lower panels. Scale bar=100 $\mu m$. B: TNXB expression by immunohistochemistry in $M M$ and $A C$. The proportion of positively stained area in $M M(n=30)$ was significantly higher than that for AC ( $n=69)$ $(* * p<0.001)$. C: Receiver operating characteristic $(R O C)$ curve for TNXB staining for the detection of MM and AC. The area under the ROC curve was 0.718. D: The pie graphs show the result of classification into MM and AC using a cutoff value of $1.4 \%$ for TNXB staining. 
out of $24 \mathrm{MM}$ cases without calretinin staining (Table I). In addition, TNXB was negative in only one out of four lung adenocarcinoma with calretinin-positive staining. When the detection of TNXB was combined with that of calretinin, $83.3 \%$ (25 out of 30 patients) of MM cases were detected. The sensitivity and specificity of the combination of TNXB and calretinin were $83.3 \%$ and $68.1 \%$, respectively (Table II). These findings suggest that TNXB might be used as a diagnostic biomarker for MM. A combination of detecting TNXB with calretinin may be applicable for the differential diagnosis of MM from lung adenocarcinoma.

\section{Discussion}

In addition to the poor prognosis of patients with MM, the difficulty of diagnosing MM is a major clinical issue (14). In particular, the differential diagnosis of $\mathrm{MM}$ from the pleural involvement of lung adenocarcinoma is still difficult. Several pleural markers including calretinin, WT-1 and D240 are available for the diagnosis of MM; however, their sensitivity and specificity are poor (15-19). Therefore, novel diagnostic markers that distinguish MM from lung adenocarcinoma need to be established for the optimal treatment of patients. In this study, we found that TNXB was likely to be involved in the carcinogenesis of MM. The TNXB protein level also served as a diagnostic marker to distinguish MM from lung adenocarcinoma.

Tenascin is an extracellular matrix protein glycoprotein and has four gene family members, TNX, tenascin-C (TNC), tenascin-W, and tenascin- $\mathrm{R}$ (20). Tenascin-C is expressed in various cancer types including cholangiocarcinoma, gastric cancer, and breast cancer (21-23). Tenascin-C was also reported to be overexpressed in $\mathrm{MM}$ and be associated with metastasis (24-25). In contrast, TNX is involved in collagen organization and matrix integrity (26). TNX has two gene copies TNXA and $T N X B$. TNXB variants have been associated with EhlersDanlos syndrome (27). A previous study reported that $T N X B$ mRNA expression was higher in MM compared with ovarian carcinoma, primary peritoneal carcinoma, and breast carcinoma (7). In this study, we found high $T N X B$ expression in MM compared with paired normal tissues as well as lung adenocarcinoma. Although the sensitivity of calretinin for detection of MM in this study was lower than in previous studies, we demonstrated that the sensitivity of TNXB was superior to the sensitivity of calretinin as an MM diagnostic marker (15-19). Furthermore, TNXB combined with calretinin improved its diagnostic ability for MM. A definitive diagnosis of MM might allow optimal treatment for patients with MM.

Pemetrexed combined with cisplatin as first-line and nivolumab as second-line treatments are recommended as standard therapies for patients with MPM; however, their effectiveness is limited $(4,5)$; therefore, new therapeutic agents are required for patients with MPM. TNXB might be both a diagnostic marker and a therapeutic target for MM. In our previous analysis using MM cell lines, lower TNXB expression tended to indicate sensitivity to pemetrexed (6). We did not determine the significance of changes in sensitivity to pemetrexed after TNXB inhibition (data not shown). However, the suppression of cell proliferation and decreased colony formation after TNXB inhibition were observed in this study, suggesting TNXB might be a novel therapeutic target.

In conclusion, we found that TNXB is a novel diagnostic marker for MM. The combined use of TNXB and calretinin may be applicable for the differential diagnosis of MM from lung adenocarcinoma. Further studies should be undertaken to evaluate the usefulness and robustness of TNXB combined with calretinin as a diagnostic marker.

\section{Conflicts of Interest}

No potential conflicts of interest are disclosed.

\section{Authors' Contributions}

Conception and design: K. Nakayama, M. Seike, R. Noro, A. Gemma. Development of methodology: K. Nakayama, S.Takeuchi, R. Noro. Acquisition of data: K. Nakayama, M. Seike. Analysis and interpretation of data (e.g. statistical analysis, biostatistics, image analysis): K. Nakayama, M. Seike, R. Noro. Writing, review, and/or revision of the manuscript: K. Nakayama, M. Seike. Administrative, technical, or material support: R. Noro, K. Matsuda, S. Kunugi. Supervisor: K. Kubota, A. Gemma.

\section{Acknowledgements}

This study was supported by a Clinical Rebiopsy Bank Project for Comprehensive Cancer Therapy Development (to M. Seike and A. Gemma). The Authors thank Ms M. Takatori for excellent technical assistance.

\section{References}

1 Robinson BW and Lake RA: Advances in malignant mesothelioma. N Engl J Med 353: 1591-1603, 2005. PMID: 16221782, DOI: 10.1056/NEJMra050152

2 Robinson BW, Musk AW and Lake RA: Malignant mesothelioma. Lancet 366: 397-408, 2005. PMID: 16054941, DOI: 10.1016/S0140-6736(05)67025-0

3 Rehrauer H, Wu L, Blum W, Pecze L, Henzi T, Serre-Beinier V, Aquino C, Vrugt B, de Perrot M, Schwaller B and Felley-Bosco E: How asbestos drives the tissue towards tumors: YAP activation, macrophage and mesothelial precursor recruitment, RNA editing, and somatic mutations. Oncogene 37: 2645-2659, 2018. PMID: 29507420, DOI: $10.1038 / \mathrm{s} 41388-018-0153-\mathrm{z}$

4 Vogelzang NJ, Rusthoven JJ, Symanowski J, Denham C, Kaukel E, Ruffie P, Gatzemeier U, Boyer M, Emri S, Manegold C, Niyikiza $\mathrm{C}$ and Paoletti P: Phase III study of pemetrexed in combination with cisplatin versus cisplatin alone in patients with malignant pleural mesothelioma. J Clin Oncol 21: 2636-2644, 2003. PMID: 12860938, DOI: 10.1200/JCO.2003.11.136 
5 Goto Y, Okada M, Kijima T, Aoe K, Kato T, Fujimoto N, Nakagawa K, Takeda Y, Hida T, Kanai K, Imamura F, Oizumi S, Takahashi T, Takenoyama M, Tanaka H and Ohe Y: A phase II study of nivolumab: a multicenter, open-label, single arm study in malignant pleural mesothelioma (MERIT). International Association for the Study of Lung Cancer 18th World Conference on Lung Cancer: Yokohama, Japan, 2017.

6 Takeuchi S, Seike M, Noro R, Soeno C, Sugano T, Zou F, Uesaka H, Nishijima N, Matsmoto M, Minegishi Y, Kubota K and Gemma A: Significance of osteopontin in the sensitivity of malignant pleural mesothelioma to pemetrexed. Int J Oncol 44: 1886-1894, 2014. PMID: 24714722, DOI: 10.3892/ ijo.2014.2370

7 Yuan Y, Nymoen DA, Stavnes HT, Rosnes AK, Bjørang O, Wu $\mathrm{C}$, Nesland JM and Davidson B: Tenascin- $\mathrm{X}$ is a novel diagnostic marker of malignant mesothelioma. Am J Surg Pathol 33: 1673-1682, 2009. PMID: 19738457, DOI: 10.1097/PAS.0b013e3181b6bde3

8 Suraokar MB, Nunez MI, Diao L, Chow CW, Kim D, Behrens C, Lin H, Lee S, Raso G, Moran C, Rice D, Mehran R, Lee JJ, Pass HI, Wang J, Momin AA, James BP, Corvalan A, Coombes $\mathrm{K}$, Tsao A and Wistuba II: Expression profiling stratifies mesothelioma tumors and signifies deregulation of spindle checkpoint pathway and microtubule network with therapeutic implications. Ann Oncol 25: 1184-1192, 2014. PMID: 24669013, DOI: $10.1093 /$ annonc/mdu127

9 Usami N, Fukui T, Kondo M, Taniguchi T, Yokoyama T, Mori S, Yokoi K, Horio Y, Shimokata K, Sekido Y and Hida T: Establishment and characterization of four malignant pleural mesothelioma cell lines from Japanese patients. Cancer Sci 97: 387-394, 2006. PMID: 16630136, DOI: 10.1111/j.13497006.2006.00184.x

10 Seike M, Goto A, Okano T, Bowman ED, Schetter AJ, Horikawa I, Mathe EA, Jen J, Yang P, Sugimura H, Gemma A, Kudoh S, Croce CM and Harris CC: $m i R-21$ is an EGFR-regulated antiapoptotic factor in lung cancer in never-smokers. Proc Natl Acad Sci USA 106: 12085-12090, 2009. PMID: 19597153, DOI: 10.1073/pnas. 0905234106

11 Nakamichi S, Seike M, Miyanaga A, Chiba M, Zou F, Takahashi A, Ishikawa A, Kunugi S, Noro R, Kubota K and Gemma A: Overcoming drug-tolerant cancer cell subpopulations showing AXL activation and epithelial-mesenchymal transition is critical in conquering ALK-positive lung cancer. Oncotarget 9: 2724227255, 2018. PMID: 29930762, DOI: 10.18632/oncotarget.25531

12 Bustin SA: Absolute quantification of mRNA using real-time reverse transcription polymerase chain reaction assays. J Mol Endocrinol 25: 169-193, 2000. PMID: 11013345, DOI: 10.1677/jme.0.0250169

13 Takahashi A, Seike M, Chiba M, Takahashi S, Nakamichi S, Matsumoto M, Takeuchi S, Minegishi Y, Noro, R, Kunugi S, Kubota $\mathrm{K}$ and Gemma A: Ankyrin repeat domain 1 overexpression is associated with common resistance to afatinib and osimertinib in EGFR-mutant lung cancer. Sci Rep 8: 14896, 2018. PMID: 30291293, DOI: 10.1038/s41598-018-33190-8

14 Taioli E, Wolf AS, Camacho-Rivera M, Kaufman A, Lee DS, Nicastri D, Rosenzweig K and Flores RM: Determinants of survival in malignant pleural mesothelioma: A Surveillance, Epidemiology, and End Results (SEER) study of 14,228 patients. PLoS One 10: e0145039, 2015. PMID: 26660351, DOI: 10.1371/journal.pone.0145039
15 Oates J and Edwards C: HBME-1, MOC-31, WT1 and calretinin: an assessment of recently described markers for mesothelioma and adenocarcinoma. Histopathology 36: 341-347, 2000. PMID: 10759948, DOI: 10.1046/j.1365-2559.2000.00770.x

16 Marchevsky AM: Application of immunohistochemistry to the diagnosis of malignant mesothelioma. Arch Pathol Lab Med 132: 397-401, 2008. PMID: 18318582, DOI: 10.1043/15432165(2008)132[397:AOITTD]2.0.CO;2

17 Ordóñez NG: The immunohistochemical diagnosis of mesothelioma: a comparative study of epithelioid mesothelioma and lung adenocarcinoma. Am J Surg Pathol 27: 1031-1051, 2003. PMID: 12883236, DOI: 10.1097/00000478-200311000-00003

18 Padgett DM, Cathro HP, Wick MR and Mills SE: Podoplanin is a better immunohistochemical marker for sarcomatoid mesothelioma than calretinin. Am J Surg Pathol 32: 123-127, 2008. PMID: 18162779, DOI: 10.1097/PAS.0b013e31814faacf

19 Washimi K, Yokose T, Yamashita M, Kageyama T, Suzuki K, Yoshihara M, Miyagi Y, Hayashi H and Tsuji S: Specific expression of human intelectin-1 in malignant pleural mesothelioma and gastrointestinal goblet cells. PLoS One 7: e39889, 2012. PMID: 22768319, DOI: 10.1371/journal.pone.0039889

20 Chiovaro F, Chiquet-Ehrismann R and Chiquet M: Transcriptional regulation of tenascin genes. Cell Adh Migr 9: 34-47, 2015. PMID: 25793574, DOI: 10.1080/19336918.2015.1008333

21 Aishima S, Taguchi K, Terashi T, Matsuura S, Shimada M and Tsuneyoshi M: Tenascin expression at the invasive front is associated with poor prognosis in intrahepatic cholangiocarcinoma. Mod Pathol 16: 1019-1027, 2003. PMID: 14559985, DOI: 10.1097/01.MP.0000086860.65672.73

22 Wiksten JP, Lundin J, Nordling S, Lundin M, Kokkola A, von Boguslawski $\mathrm{K}$ and Haglund C: Tenascin-C expression correlates with prognosis in gastric cancer. Oncology 64: 245-250, 2003. PMID: 12697965, DOI: 10.1159/000069305

23 Oskarsson T, Acharyya S, Zhang XH, Vanharanta S, Tavazoie SF, Morris PG, Downey RJ, Manova-Todorova K, Brogi E and Massagué $\mathrm{J}$ : Breast cancer cells produce tenascin $\mathrm{C}$ as a metastatic niche component to colonize the lungs. Nat Med 17: 867-874, 2011. PMID: 21706029, DOI: 10.1038/nm.2379

24 Kaarteenaho-Wiik R, Soini Y, Pöllänen R, Pääkkö P and Kinnula VL: Overexpression of tenascin-C in malignant pleural mesothelioma. Histopathology 42: 280-291, 2003. PMID: 12605648, DOI: 10.1046/j.1365-2559.2003.01568.x

25 Lowy CM and Oskarsson T: Tenascin C in metastasis: A view from the invasive front. Cell Adh Migr 9: 112-124, 2015. PMID: 25738825, DOI: $10.1080 / 19336918.2015 .1008331$

26 Egging D, van Vlijmen-Willems I, van Tongeren T, Schalkwijk J and Peeters A: Wound healing in tenascin- $X$ deficient mice suggests that tenascin- $X$ is involved in matrix maturation rather than matrix deposition. Connect Tissue Res 48: 93-98, 2007. PMID: 17453911, DOI: 10.1080/03008200601166160

27 Schalkwijk J, Zweers MC, Steijlen PM, Dean WB, Taylor G, van Vlijmen IM, van Haren B, Miller WL, Bristow J: A recessive form of the Ehlers-Danlos syndrome caused by tenascin-X deficiency. N Engl J Med 345: 1167-1175, 2001. PMID: 11642233, DOI: 10.1056/NEJMoa002939

Received December 29, 2018

Revised January 19, 2019

Accepted January 21, 2019 